\title{
Are YouTube Videos in Turkish Useful as an Information Source for Dental Treatment During the COVID-19 Pandemic?
}

Gözde Işık

University of Ege,Izmir, gozdech@hotmail.com

Meltem Özden Yüce

University of Ege,izmir, meltemozdn@hotmail.com

Follow this and additional works at: https://nsuworks.nova.edu/ijahsp

Part of the Oral and Maxillofacial Surgery Commons

\section{Recommended Citation}

Işık G, Özden Yüce M. Are YouTube Videos in Turkish Useful as an Information Source for Dental Treatment During the COVID-19 Pandemic?. The Internet Journal of Allied Health Sciences and Practice. 2021 Jan 01;19(2), Article 18.

This Manuscript is brought to you for free and open access by the College of Health Care Sciences at NSUWorks. It has been accepted for inclusion in Internet Journal of Allied Health Sciences and Practice by an authorized editor of NSUWorks. For more information, please contact nsuworks@nova.edu. 


\title{
Are YouTube Videos in Turkish Useful as an Information Source for Dental Treatment During the COVID-19 Pandemic?
}

\begin{abstract}
Purpose: Since the start of the coronavirus disease-19 (COVID-19) pandemic, patients with dental conditions find it difficult to consult their physicians and to preserve the continuity of dental treatment. All routine appointments were suspended and postponed indefinitely so that infection control procedures could be administered. This study aimed to assess the value of Turkish videos for patients seeking information on dental treatments during the COVID-19 outbreak. Methods: Videos on YouTube were screened using the following keywords: 'Coronavirus, diş tedavileri', 'COVID-19, diş tedavileri' and 'SARSCov-2, diş tedavileri'. The search results were limited to the first 100 videos. Videos were analysed independently by two examiners and re-evaluated by an independent examiner. The scope was limited to videos in Turkish. Results: The keyword search resulted in an initial list of 919 videos, from which the first 100 videos were viewed. The final analysis included 39 videos that qualified the inclusion criteria. Of the 39 videos, only one had excellent quality, while 13 (33.3\%) had poor quality. Conclusion: Our study demonstrates that YouTube videos in the Turkish language were limited sources for patients seeking reliable information on dental treatments during the COVID-19 pandemic. Further studies from other countries are needed to evaluate the available YouTube videos during and after the period of the COVID-19 outbreak.
\end{abstract}

\section{Author Bio(s)}

Gözde Işık, is a Lecturer of Oral and Maxillofacial Surgery Department at School of Dentistry, Ege University.

Meltem Özden Yüce, is PhD of Oral and Maxillofacial Surgery. She is working as a research assistant in Oral Surgery Department of School of Dentistry, Ege University.

\section{Acknowledgements}

The authors are greatful to Dr. Emine ADALI from İzmir Demokrasi University, for her extraordinary support by re-evaluating the videos in this study. 


\title{
IIJAHSP
}

\section{The Internet Journal of Allied Health Sciences and Practice \\ Dedicated to allied health professional practice and education}

Vol. 19 No. 2 ISSN 1540-580X

\section{Are YouTube Videos in Turkish Useful as an Information Source for Dental Treatment During the COVID-19 Pandemic?}

\author{
Gözde Işık \\ Meltem Özden Yüce \\ University of Ege \\ İmir, Turkey
}

\begin{abstract}
Purpose: Since the start of the coronavirus disease-19 (COVID-19) pandemic, patients with dental conditions find it difficult to consult their physicians and to preserve the continuity of dental treatment. All routine appointments were suspended and postponed indefinitely so that infection control procedures could be administered. This study aimed to assess the value of Turkish videos for patients seeking information on dental treatments during the COVID-19 outbreak. Methods: Videos on YouTube were screened using the following keywords: 'Coronavirus, diş tedavileri', 'COVID-19, diş tedavileri' and 'SARS-Cov-2, diş tedavileri'. The search results were limited to the first 100 videos. Videos were analysed independently by two examiners and re-evaluated by an independent examiner. The scope was limited to videos in Turkish. Results: The keyword search resulted in an initial list of 919 videos, from which the first 100 videos were viewed. The final analysis included 39 videos that qualified the inclusion criteria. Of the 39 videos, only one had excellent quality, while $13(33.3 \%)$ had poor quality. Conclusion: Our study demonstrates that YouTube videos in the Turkish language were limited sources for patients seeking reliable information on dental treatments during the COVID19 pandemic. Further studies from other countries are needed to evaluate the available YouTube videos during and after the period of the COVID-19 outbreak.
\end{abstract}

Keywords: COVID-19, dental treatment, disease outbreaks 


\section{INTRODUCTION}

The outbreak of a novel viral infection, referred to as coronavirus disease 2019 (COVID-19), was first reported in Wuhan, China, on 31 December 2019. ${ }^{1}$ Following the rapid spread of COVID-19 to other provinces in China and to countries worldwide, ${ }^{2}$ the World Health Organization (WHO) declared this disease as a 'public health emergency of international concern' on 30 January 2020.3 The severe acute respiratory syndrome coronavirus is transmitted through direct or close contact with body fluids or blood and droplets from the respiratory tract of patients with COVID-19 infection. Given the clear necessity for close proximity to patients' oral and nasal cavities, dentistry carries a high potential risk for transmission of COVID-19 in the course of routine clinical practice. ${ }^{1-5}$

In Turkey, the first COVID-19 case was announced on 11 March 2020, and from the first day, strict rules were introduced to control the transmission of COVID-19, including home isolation, quarantine, as well as restrictions on travel and transportation. ${ }^{6}$ Dental hospital clinics and academic institutions were reorganised to concentrate resources on key emergencies involving trauma, infections or malignant neoplasm. Furthermore, all routine appointments were suspended and postponed indefinitely so that infection control procedures could be administered. ${ }^{7}$ As a consequence, it has become very difficult for patients to consult their physicians and to preserve the continuity of dental treatment during the COVID-19 pandemic.

On 18 March 2020, the American Dental Association developed a guideline about dental emergency and nonemergency care. In this guideline, patients with dental concerns were categorised according to the urgency and severity of the dental interventions. Patients with uncontrolled bleeding, dentoalveolar trauma or progressive orofacial infection were specified to require emergency dental intervention; however, aesthetic and restorative dentistry procedures were classified as nonemergency dental procedures. Therefore, during the COVID-19 pandemic, elective/routine dental treatments have been contraindicated. Accurate information including dental disease classification based on the degree of emergency that is shared or published may have a positive effect on patients with dental concerns. ${ }^{8,9}$

Previous studies have reported that online platforms are commonly used by patients as sources of health-related information. 10-13 YouTube, as one of these sources, is ranked as the second most visited website across all countries, with more than one billion users worldwide. ${ }^{14}$ It allows visitors to view and upload free videos through the use of specific search terms. ${ }^{13}$ YouTube provides easy access to information through its vast range of videos and clearly has huge potential to influence its viewers. ${ }^{12}$ However, it has no regulatory policy to ensure the factual accuracy of content; therefore, video quality varies, with no filtering of misleading information. For this reason, it is essential to evaluate the effectiveness of YouTube videos as an information source for patients.

This study aimed to assess the value of Turkish videos for patients with dental concerns seeking information about COVID-19, transmission-based precautions in dental practice and conditions that should be referred to a dental health professional during a pandemic.

\section{METHODS}

This cross-sectional study was conducted on 26 July 2020 between 8:30 a.m. and 17:30 p.m. As this study was carried out through the analysis of publicly available information, institutional review board approval was not required.

Videos on YouTube (www.YouTube.com) were screened for information related to the main study topic using the following keywords: 'Coronavirus, diş tedavileri', 'COVID-19, diş tedavileri' and 'SARS-Cov-2, diş tedavileri'. 'Diş tedavileri' is the Turkish translation for dental treatments. The inclusion criteria of the study were videos in the Turkish language with acceptable visual and audio quality. The exclusion criteria were as follows: 1) irrelevant to the main study topic, 2) non-Turkish videos, 3) dental health professionals as target users, 4) duplicates, 5) advertisements, 6) videos giving dental practices' working hours, 7) lack of visual and audio quality and 8 ) video running time $<2 \mathrm{~min}$ and $>15 \mathrm{~min} .15$

\section{Video Assessment}

Videos were analysed independently by two examiners ( $\mathrm{Gl}$ and MOY). Prior to the analysis, cookies and previous searches were deleted. YouTube viewers usually click no further than the first three pages of the search results, and only those who cannot find the required information on the first page proceed to other pages ${ }^{16}$ Therefore, the search results were limited to the first 100 videos. The search results were recorded by creating a playlist to avoid variations in the examiners' analyses. Furthermore, all videos were re-evaluated by an independent examiner to confirm data validity and reliability.

\section{Assessment of Video Parameters}

Basic information details and variables were collected for each video, including upload date, running time, total number of views and total 'likes', 'dislikes' and comments. Viewers' interactions were calculated using the interaction index ([number of likes number of dislikes] / total number of views $\times 100 \%$ ) and the viewing rate (number of views/number of days since upload $\times 100 \%$ ), which were identified previously. ${ }^{11}$ 
Videos were also classified into five categories according to the source of the upload: dental practice committees, dental health centres, dental health professionals, non-professional users and news and information websites.

\section{Assessment of Quality}

The Global Quality Scale (GQS) ${ }^{17}$ was used to assess the quality of each video according to the extent of its content of scientifically accurate information about dental treatments during the COVID-19 outbreak. The following five aspects of the content of the videos were used for evaluation: 1) characteristics of COVID-19, 2) transmission routes of COVID-19 in dental practice, 3) conditions that should be referred to a dental health professional during the pandemic, 4) triage and treatment priorities in dental practice and 5) transmission-based precautions in the care of patients [4]. These aspects of the content were assigned points from 1 to 5 , and the total score was incorporated into the GQS score.

To calculate the flow, usefulness and overall quality of videos, a five-point Likert-type GQS score was used, as shown below:

1. Poor quality, poor flow and not helpful to patients

2. Generally of poor quality, poor flow, presented some information and limited use to patients

3. Moderate quality, suboptimal flow, presented some important information, did not cover other important topics and useful to some extent for patients

4. Good quality, good flow, covered most important topics, useful for patients, but videos may include minor deficiencies.

5. Excellent quality, excellent flow, presented detailed, valid, and accurate information and very useful to patients.

\section{Assessment of Reliability}

The modified DISCERN instrument was used to assess the reliability of the content in each video. ${ }^{18}$ This questionnaire comprised the following five questions with 'yes' or 'no' answers: 1) Are the aims clear and achieved? 2) Are reliable sources of information used (e.g., highly regarded publications, cited, and qualified dental healthcare professionals featured)? 3) Is the information presented both balanced and unbiased? 4) Are additional sources of information listed for patient reference? 5) Are areas of uncertainty mentioned? Each question was assigned 1 point for an answer of 'yes' and 0 points for ' $n o$ '. The total score ranged from 1 to 5 points.

\section{Data Analysis}

Statistical analysis was carried out using the IBM SPSS Statistics 25.0 software programme (IBM Corp., Armonk, NY). Descriptive statistics were calculated for each variable as mean, standard deviations (median, minimum and maximum), frequency and percentage. The inter-observer agreement was calculated as a kappa score. Quantitative variables were tested for normality using the Shapiro-Wilk test. If variables were normally distributed, the one-way analysis of variance test and Bonferroni correction were used. Comparisons of more than two non-normally distributed variables were evaluated using the Kruskal-Wallis test. Correlations were evaluated using Spearman's rho correlation coefficient. The statistical significance level was set at $p<0.05$.

\section{RESULTS}

The keyword search resulted in an initial list of 919 videos, from which the first 100 videos were viewed. After the initial screening, many videos were excluded in line with the exclusion criteria: 22 videos were considered not relevant to the main study topic, four videos were duplicates, 14 videos were $<2$ min and 21 videos were $>15$ min. Finally, the remaining 39 videos were qualified for inclusion in this study. The overall inter-examiner agreement calculated as a weighted kappa score was $0.79(p<0.001)$.

The largest proportions of videos have been uploaded by dental healthcare professionals ( $46.2 \%)$, followed by dental health centres $(35.9 \%)$ and news websites (17.9\%). The mean interaction index and mean viewing rates were $3.34 \% \pm 4.22 \%$ and $0.18 \% \pm 0.79 \%$, respectively. Moreover, the mean scores were $1.89 \pm 0.77$ for GQS and $0.44 \pm 0.26$ for the modified DISCERN. The characteristics of the videos are summarised in Table 1.

Table 1: Characteristics of videos included for analysis.

\begin{tabular}{l|l|l}
\hline \multicolumn{2}{c}{ Mean + SD } & Median (Min-Max) \\
\hline Number of days & $89.25 \pm 29.50$ & $86(3-131)$ \\
\hline Number of comments & $3.05 \pm 12.52$ & $0(0-76)$ \\
\hline Number of views & $1708.07 \pm 8232.88$ & $206(12-51699)$ \\
\hline
\end{tabular}

(C) The Internet Journal of Allied Health Sciences and Practice, 2021 


\begin{tabular}{l|l|l}
\hline Number of likes & $30.61 \pm 141.60$ & $7(0-891)$ \\
\hline Number of dislikes & $1.82 \pm 7.07$ & $0(0-44)$ \\
\hline Running time of video (min) & $5.30 \pm 3.02$ & $4.5(2-12.0)$ \\
\hline Interaction index & $3.34 \pm 4.22$ & $1.6(0-15.8)$ \\
\hline Viewing rate & $0.18 \pm 0.79$ & $0.02(0.00-4.97)$ \\
\hline Mean GQS scores & $1.89 \pm 0.77$ & $1.8(1-4.8)$ \\
\hline Mean modified DISCERN score & $0.44 \pm 0.26$ & $0.4(0-1)$ \\
\hline
\end{tabular}

SD: Standard Deviation

The GQS analysis determined that $>80 \%$ of the videos had poor quality with regard to two aspects of their content: 'characteristics of COVID-19' and 'triage and treatment priorities in dental practice'. As regards the quality of the videos, $13(33.3 \%)$ had poor quality, $19(48.7 \%)$ had generally poor quality, five $(12.8 \%)$ had moderate quality, one $(2.6 \%)$ had good quality and one $(2.6 \%)$ had excellent quality (Table 2).

Table 2: Quality of analyzed videos

\begin{tabular}{|c|l|l|l|l|l|l|l|}
\hline & Poor quality & $\begin{array}{l}\text { Generally } \\
\text { poor quality }\end{array}$ & $\begin{array}{l}\text { Moderate } \\
\text { quality }\end{array}$ & $\begin{array}{l}\text { Generally } \\
\text { good quality }\end{array}$ & Good quality & Total \\
\hline $\begin{array}{c}\text { Analyzed } \\
\text { videos }\end{array}$ & Frequency & 13 & 19 & 5 & 1 & 1 & 39 \\
\cline { 2 - 8 } & Percent (\%) & 33.3 & 48.7 & 12.8 & 2.6 & 2.6 & 100.0 \\
\hline
\end{tabular}

Seven videos (100\%) uploaded by news websites and $13(92.8 \%)$ by dental health centres had low-quality, while only two videos $(11.2 \%)$ uploaded by dental health professionals had high-quality. The mean GQS score of the videos uploaded by dental health professionals was higher than that of videos uploaded by dental health centres and news websites. The quality difference between videos from different sources was statistically significant $(p=0.031)$. The comparison of GQS scores according to the upload source is presented in Table 3.

Table 3: Comparison of General Quality Scale according to the source of upload. Data were expressed as Mean \pm SD

\begin{tabular}{|l|l|l|l|l|}
\hline & $\begin{array}{l}\text { Dental health } \\
\text { centers } \\
(\mathbf{n = 1 4 )}\end{array}$ & $\begin{array}{l}\text { Dental health } \\
\text { professionals } \\
(\mathbf{n = 1 8 )}\end{array}$ & $\begin{array}{l}\text { News } \\
(\mathbf{n}=7)\end{array}$ & p \\
\hline GQS (General Quality Scale) & $1.72 \pm 0.50$ & $2.22 \pm 0.92 \dagger$ & $1.40 \pm 0.40$ & $0.031^{*}$ \\
\hline
\end{tabular}

One-way ANOVA test: * $\mathrm{p}<0.05$ significant difference between groups.

Pairwise comparisons with Bonferroni correction: ${ }^{\dagger} \mathrm{p}<0.016$ significantly higher than News.

When the contents of the videos were compared, based on the GQS scores between video sources, a significant difference was found between the sources by 'characteristics of COVID-19' score $(p=0.018)$. Although the median score of 'transmission routes of COVID-19 in dental practice' was higher in videos uploaded by dental health professionals than those by dental health centres and news websites, the difference was not significant $(p=0.090)$. Moreover, for 'transmission-based precautions in the care of patients', the median scores of the videos uploaded by dental health professionals and dental health centres were higher than those 
for by news websites. However, the difference was not significant $(p=0.135)$. The content analysis of the videos based on $G Q S$ scores is presented in Table 4.

Table 4: Detailed content analysis of videos based on General Quality Scale (GQS) scores. Data were expressed as Median (Min-Max)

\begin{tabular}{|c|c|c|c|c|}
\hline 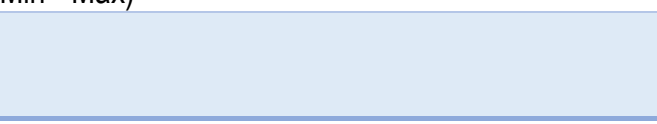 & $\begin{array}{l}\text { Dental health } \\
\text { centers } \\
(n=14)\end{array}$ & $\begin{array}{l}\text { Dental health } \\
\text { professionals } \\
(n=18)\end{array}$ & $\begin{array}{l}\text { News } \\
(n=7)\end{array}$ & $\mathbf{p}$ \\
\hline Characteristics of COVID-19 & $1.0(1.0-1.0)$ & $1.0(1.0-5.0)^{\dagger}$ & $1.0(1.0-1.0)$ & 0.018 \\
\hline Transmission routes of COVID-19 in dental practice & $1.0(1.0-3.0)$ & $2.0(1.0-5.0)$ & $1.0(1.0-3.0)$ & 0.090 \\
\hline $\begin{array}{l}\text { Conditions that should be referred to a dental health } \\
\text { professional during the pandemic }\end{array}$ & $1.0(1.0-5.0)$ & $1.0(1.0-5.0)$ & $1.0(1.0-4.0)$ & 0.722 \\
\hline Triage and treatment priorities in dental practice & $1.0(1.0-3.0)$ & $1.0(1.0-5.0)$ & $1.0(1.0-1.0)$ & 0.231 \\
\hline $\begin{array}{l}\text { Transmission-based precautions in the care of } \\
\text { patients }\end{array}$ & $3.0(1.0-5.0)$ & $3.5(1.0-5.0)$ & $1.0(1.0-5.0)$ & 0.135 \\
\hline
\end{tabular}

Kruskal-Wallis test: ${ }^{*} \mathrm{p}<0.05$ significant difference between groups.

Dunn's test with Bonferroni correction: ${ }^{\dagger} \mathrm{p}<0.016$ significantly higher than Dental health centers.

In the analysis of the reliability of the videos, the mean modified DISCERN scores were low for each video source $(0.44 \pm 0.23$ for dental health centres, $0.52 \pm 0.30$ for dental healthcare professionals and $0.25 \pm 0.09$ for news websites). For all questions, the scores of videos uploaded by news websites and dental health centres were low compared with those by dental health professionals. However, the differences were not significant $(p=0.078)$. The comparison of modified DISCERN scores, by source of upload, is presented in Table 5.

Table 5: Comparison of DISCERN scores according to the source of upload. Data were expressed as Mean \pm SD

\begin{tabular}{|l|l|l|l|l|}
\hline & $\begin{array}{l}\text { Dental health } \\
\text { centers } \\
(\mathbf{n = 1 4 )}\end{array}$ & $\begin{array}{l}\text { Dental health } \\
\text { professionals } \\
(\mathbf{n = 1 8 )}\end{array}$ & $\begin{array}{l}\text { News } \\
(\mathbf{n}=7)\end{array}$ & $\mathbf{p}$ \\
\hline Mean modified DISCERN score & $0.44 \pm 0.23$ & $0.52 \pm 0.30$ & $0.25 \pm 0.09$ & 0.078 \\
\hline
\end{tabular}

One-way ANOVA test

Spearman's rho correlation analysis showed a positive correlation between the mean GQS and mean modified DISCERN scores $(r=0.591 ; p<0.001)$. A significant correlation was found between the mean GQS scores and the 'like' and 'comment' counts $(r=$ $0.323, p=0.045 ; r=0.404, p=0.011$, respectively). However, according to the mean GQS and mean modified DISCERN scores, no significant correlations were found between these scores and number of views, interaction index and viewing rate $(p>0.05)$.

\section{DISCUSSION}

Since the start of the COVID-19 pandemic, patients have difficulty obtaining advice and information about dental issues and, for existing patients, to preserve the continuity of treatment. For some, online platforms such as YouTube are considered a convenient means to access relevant information. ${ }^{6,10-13,19} \mathrm{As}$ a free online video-sharing platform, YouTube provides has the potential to make available a range of detailed information concerning COVID-1919-21 and dental treatments. ${ }^{10-13}$ Despite this potential, the quality and reliability of the information provided in YouTube videos highly vary and is therefore an important topic for research. ${ }^{22}$ In this study, we evaluated the usefulness of Turkish language videos for patients seeking information on dental treatments during the COVID19 pandemic. Our results demonstrated that YouTube videos in the Turkish language were insufficient sources of information. Studies from other countries can be useful, as they help researchers understand how content is presented in different languages in YouTube videos ${ }^{6}$ and how patients of different nationalities search these online platforms for health-related information. ${ }^{23}$ 
Therefore, our study could provide a useful resource for researchers and could be used as a sample design for studies in other languages.

COVID-19 has proved to be a major threat to global public health, affecting almost every country, with over 2.5 million confirmed cases and over 170.000 reported deaths. ${ }^{24,25}$ The virus spreads easily through respiratory droplets, by direct or indirect contact.1.5 Therefore, people should have access to accurate information on how to avoid contracting the virus and how to prevent transmission. ${ }^{25}$

Given its wide-ranging reach, YouTube can be a powerful resource in promoting understanding of the behaviour of COVID-19.20 Previous studies have examined the information content of YouTube videos about the emerging virus, including such aspects as its key characteristics, transmission pathways and prevention methods. ${ }^{20,21,26}$ D'Souza et al. ${ }^{21}$ reported that even if videos are useful, the videos generally lacked a comprehensive medical content on COVID-19, particularly with regard to details of transmission pathways. Similarly, in our study, two key aspects of the content, i.e. 'characteristics of COVID-19' and 'transmission routes of COVID-19 in dental practice', were found to be little discussed in videos.

Several studies have evaluated YouTube videos for their content of various health-related topics, including dental health. ${ }^{10-13,22,23}$ Menziletoğlu et al. ${ }^{12}$ and Nason et al..$^{13}$ reported that the majority of the videos included were not very extensive in their coverage of the subjects, and most of the important parameters were not mentioned. Similarly, Ho et al. ${ }^{10}$ reported that the videos contained insufficient information to achieve the intended educational objectives. In our study, the content of the videos was not effective in conveying worthwhile information about dental treatments during COVID-19. With regard to the promotion of valid and credible information, several studies have analysed the correlation between the sources of the uploaded videos and content quality. The quality of the videos varied significantly by source and content. 13,23,27 Similarly, our study results showed that the mean quality scores of the videos uploaded by the dental health professionals were higher than those of the dental health centres and news websites. Moreover, these videos contained more information on the 'characteristics of COVID-19' and 'transmission routes of COVID-19 in dental practice' than videos by the dental health centres and news. Therefore, dental specialists and practitioners should recognise YouTube as a significant platform for the spread of useful information, particularly in regard to the COVID-19 pandemic. In this way, the availability of high-quality and reliable factual information with evidence-based data will lead to betterinformed patients.

The transmission risk of the COVID-19 virus is high in dental interventions, as in most cases they involve aerosol generation and require proximity of dental professionals to the patient's oropharyngeal region. ${ }^{1-4}$ Therefore, treatment priorities had to be re-defined according to the degree of urgency in dental practices, taking account transmission risk. ${ }^{4}$ However, we found that most of the videos lacked any useful content related to the 'conditions that should be referred to a dental health professional during the pandemic' and 'triage and treatment priorities in dental practice'. Distinction between interventions of urgent and intermediate priority were not clearly explained in YouTube videos. They are not effective enough and therefore should provide comprehensive information for patients about elective and emergency procedures in dental practice.

Nevertheless, with regard to the content, videos uploaded by dental health professionals and dental health centres presented more information than those uploaded by news websites, including 'transmission-based precautions in the care of patients'. A practical approach to reducing the transmission risk of COVID-19 may be to allocate more educational resources to prevent transmission.,3,4 Therefore, being aware of currently available information and providing patients with more accurate content are important.

Many studies have taken into account the total number of views of the videos and evaluated them in terms of content quality and reliability.23,27 Some studies reported that the poor quality and reliability of the information in YouTube videos might result in low view rate. ${ }^{27}$ Similar to results of Gaş et al., ${ }^{23}$ our results showed that low-quality video, including poor or misleading information, did not result in a reduced number of views by patients. In turn, a relatively high-quality video, in terms of the amount of useful content, did not correlate with a high viewing rate. Therefore, the predominance of low-quality content in YouTube videos might result in the widespread dissemination of misleading information, limiting opportunities for people to receive correct information about dental treatments under COVID-19 conditions. Despite concerns about misleading information, YouTube videos will continue to be seen as an easily accessible source of information for patients. ${ }^{13}$ Although it is not possible to prevent public access to online platforms, patients should be directed to appropriate sources to minimise the amount of inaccurate information in circulation. ${ }^{13,23}$

\section{Limitations}

Study limitations need to be taken into consideration. Published studies on YouTube videos are mostly cross-sectional, although YouTube is a dynamic media platform with videos being added every minute. Therefore, results might vary depending on the date and time of search. Moreover, the initial keywords selected are likely to significantly affect the conclusions reached. 


\section{CONCLUSION}

YouTube videos included in this study provide insufficient information on the COVID-19 pandemic; on when it is advisable to consult a dental health professional, and on treatment priorities and prevention methods in dental practice. In conclusion, our study demonstrated that YouTube videos in the Turkish language were inadequate sources of evidence for patients seeking reliable information on dental treatments during the COVID-19 pandemic. Further studies from other countries are needed to evaluate the available YouTube videos during and after the period of the COVID-19 outbreak.

\section{REFERENCES}

1. Fini MB. What dentists need to know about COVID-19? Oral Oncol. 2020;105:104741. [PMID 32380453]

2. Barca I, Cordaro R, Kallaverja E, Ferragina F, Cristofaro MG. Management in oral and maxillofacial surgery during the COVID-19 pandemic: Our experience. Br J Maxillofac Surg. 2020;58(6):687-691. [PMID 32386671]

3. Zhao Z, Gao D. Precaution of 2019 novel coronavirus infection in department of oral and maxillofacial surgery. $\mathrm{Br} J \mathrm{Oral}$ Maxillofac Surg. 2020;58:250-253. [PMID 32336398]

4. Zimmermann M, Nkenke E. Approaches to the management of patients in oral and maxillofacial surgery during COVID19 pandemic. J Cranimaxillofac Surg. 2020;48(5):521-526. [PMID 32303420]

5. Panesar K, Dodson T, Lynch J, Bryson-Cahn C, Chew L, Dillon J. Evolution of COVID-19 Guidelines for University of Washington Oral and Maxillofacial Surgery Patient Care. J Oral Maxillofac Surg. 2020;78(7):1136-1146. [PMID 32389541]

6. Yuksel B, Cakmak K. Healthcare information on YouTube: Pregnancy and COVID-19. Int J Gynaecol Obstet. 2020;150(2):189-193. [PMID 32471005]

7. Duruk G, Gümüşboğa ZŞ, Çolak C. Investigation of Turkish dentists' clinical attitudes and behaviors towards the COVID19 pandemic: a survey study. Braz Oral Res. 2020;34:054. [PMID 32490887]

8. ADA recommending dentists postpone elective procedures. Avaliable at: https://www.ada.org/en/publications/adanews/2020-archieve/march/ada-recommending-dentists-postpone-elective-procedures.Accessed March 18,2020.

9. ADA.What constitutes a dental emergency? Avaliable at: https://success.ada.org/ /media/CPS/Files/Open\%20Files/ADA COVID19 Dental Emergency DDS.pdf.Accessed April 2,2020

10. Ho A, McGrath C, Mattheos N. Social media patient testimonials in implant dentistry: information or misinformation? Clin Oral Implants Res. 2017;28(7):791-800. [PMID 27279455]

11. Abukaraky A, Hamdan AA, Ameera MN, Nasief M, Hassona Y. Quality of YouTube TM videos on dental implants. Med Oral Patol Oral Cir Bucal. 2018;23(4):463-468. [PMID 29924766]

12. Menziletoglu D, Guler AY, Isik BK. Are YouTube videos related to dental implant useful for patient education? J Stomatol Oral Maxillofac Surg. 2020; 121:661-664. [PMID 32045688]

13. Nason K, Donnelly A, Duncan HF. YouTube as a patient information source for root canal treatment. Int Endod J. 2016;49(12):1194-1200. [PMID 26551481]

14. Alexa. YouTube.com Traffic Statistics 2019. http://wwwalexacom/siteinfo/YouTube. Accessed May 13.

15. Study YSRF. https://www.briggsby.com/reverse-engineering-YouTube-search.

16. Fox S, Duggan M. Health Online (2013) Part one: Information triage.

17. Kocyigit BF, Akaltun MS, Sahin AR. YouTube as a source of information on COVID-19 and rheumatic disease link. Clin Rheumatol. 2020;39:2049-2054. [PMID 32447603]

18. Radonjic A, Fat Hing NN, Harlock J, Naji F. YouTube as source of patient information on abdominal aortic aneurysms. J Vasc Surg. 2020;71(2):637-644. [PMID 31611104]

19. Hernández-García I, Giménez-Júlvez T. Characteristics of YouTube Videos in Spanish on How to Prevent COVID-19. Int J Environ Res Public Health. 2020;17(13):4671. [PMID 32610523]

20. Basch CE, Basch CH, Hillyer GC, Jaime C. The Role of YouTube and the Entertainment Industry in Saving Lives by Educating and Mobilizing the Public to Adopt Behaviors for Community Mitigation of COVID-19: Successive Sampling Design Study. JMIR Public Health Surveill. 2020;6(2):19145. [PMID 32297593]

21. D'Souza RS, D'Souza S, Strand N, Anderson A, Vogt MNP, Olatoye O. YouTube as a source of medical information on the novel coronavirus 2019 disease (COVID-19) pandemic. Glob Public Health. 2020;15(7):935-942. [PMID 32397870]

22. Duman C. YouTube quality as a source for parent education about the oral hygiene of children. Int $J$ Dent Hyg. 2020;18(3):261-267. [PMID 32416034]

23. Gas S, Zincir ÖO, Bozkurt AP. Are YouTube Videos Useful for Patients Interested in Botulinum Toxin for Bruxism? J Oral Maxillofac Surg. 2019;77(9):1776-1783. [PMID 31077674]

24. Nicolaa M, O'Neillb N, Sohrabib C, Khanc M, Aghad M, Agha R. Evidence based management guideline for the COVID19 pandemic-Review article. Int J Surg. 2020;77:206-216. [PMID 32289472] 
25. Cevik M, Bamford CGG, Ho A. COVID-19 pandemic a focused review for clinicians. Clin Microbiol Infect.2020;26(7):842847. [PMID 32344166]

26. Basch CH, Hillyer GC, Meleo-Erwin ZC, Jaime C, Mohlman J, Basch CE. Preventive Behaviors Conveyed on YouTube to Mitigate Transmission of COVID-19: Cross-Sectional Study. JMIR Public Health Surveill. 2020; 2;6(2): e18807. [PMID 32240096]

27. Tang L, Bie B, Park SE, Zhi D. Social media and outbreaks of emerging infectious diseases: A systematic review of literature. Am J Infect Control. 2018;46(9):962-972. [PMID 29628293] 